\title{
Clonal Upregulation of Effector Cytotoxic T Lymphocytes in a Patient with Multiple Tyrosine Kinase Inhibitor-Refractory Chronic Myeloid Leukemia with Long-Term Survival
}

\author{
Tatsuro Jo ${ }^{a}$ Takahiro Sakai $^{b}$ Kaori Matsuzakab Kazuhiro Noguchib \\ Shizuka Hayashi ${ }^{b}$ Masatoshi Matsuo ${ }^{a}$ Jun Taguchi ${ }^{a}$ \\ aDepartment of Hematology, Japanese Red Cross Nagasaki Genbaku Hospital, Nagasaki, \\ Japan; 'bepartment of Laboratory, Japanese Red Cross Nagasaki Genbaku Hospital, \\ Nagasaki, Japan
}

\section{Keywords}

Chronic myeloid leukemia $\cdot$ Cytotoxic T lymphocyte $\cdot$ Tyrosine kinase inhibitor $\cdot$ T-cell receptor repertoire

\begin{abstract}
We present the case of a patient with multiple tyrosine kinase inhibitor (TKI)-refractory chronic phase chronic myeloid leukemia (CP-CML) with a T315I mutation of abl1. Dasatinib, a second-generation TKI, was administered as the initial treatment but achieved neither a cytogenetic nor molecular response. A mutational analysis of $a b l 1$ revealed that the patient had a T315I mutation. The patient was then administered ponatinib, a third-generation TKI, which is thought to be effective against T315l; however, the complete blood counts became within normal limits, and neither a cytogenetic nor molecular response was achieved. However, the patient has maintained a healthy chronic phase (with no blast crises) for more than $51 / 2$ years since the diagnosis of CP-CML. T-cell receptor (TCR) repertoire analyses using peripheral blood revealed a remarkable clonal expansion of effector cytotoxic T lymphocytes (CTLs) that contained TCR $V$ beta 13.6. We observed the clonal expansion of naïve CTLs with TCR $V$ beta 13.6; however, no clonality was observed in the memory CTLs. The naïve and effector CTLS persisted at very high percentages since the seventh month after starting dasatinib. The CTLS could not have led to the molecular response; therefore, there might be plenty of CML stem cells remaining in the bone marrow. Therefore, although the CTLs might have prevented the disease from developing blast crises over more than 5 years, the CTLs might not have been able to become memory CTLs.




\section{Introduction}

The formation of bcr-abl1 by chromosomal translocation between chromosomes 9 and 22 results in chronic myeloid leukemia (CML) [1]. Since the approval in 2001 of imatinib, the first-generation tyrosine kinase inhibitor (TKI), TKIs (including second-generation TKIs) have led to significantly improved outcomes for patients with CML [2]. However, bcr-abl1 mutations, such as T315I, have become the focus when treating patients with CML with TKIs [3-7]. Ponatinib, a third-generation TKI, has been approved for refractory CML and relapse/ refractory Philadelphia chromosome (Ph1)-positive acute lymphoblastic leukemia and has the potential for treating patients with T315I [8]. This is a case report of a patient with dasatinib and ponatinib-refractory chronic phase CML (CP-CML) with T315I who presented long-term survival with no blast crisis. The patient developed clonal naïve and effector cytotoxic T lymphocytes (CTLs) that might have prevented the patient from experiencing a blast crisis.

\section{Case Report/Case Presentation}

A 58-year-old man presented to our hospital with leukocytosis. Table 1 summarizes the patient's laboratory data. The patient had an elevated leukocyte count $(298,400 / \mu \mathrm{L})$ with 8.5\% myeloblasts, and anemia (hemoglobin, $7.5 \mathrm{~g} / \mathrm{dL}$ ). A flow cytometric analysis targeting the stem cell fraction revealed that the myeloblasts were positive for CD7, CD13, CD33, CD34, CD117, and HLA-DR, and negative for CD2, CD3, CD4, CD8, CD56, CD10, CD19, CD20, and CD235a. The results of the bone marrow aspiration were not available due to a dry tap. We detected a chromosomal abnormality $\mathrm{t}(8 ; 9 ; 22)(\mathrm{q} 22 ; \mathrm{q} 34 ; \mathrm{q} 11.2)$ in all 20 analyzed mitotic cells in a peripheral blood sample. A real-time quantitative polymerase chain reaction analysis revealed that the patient was positive for major bcr-abl1 but negative for minor bcr-abl1. A computed tomography scan revealed marked hepatosplenomegaly (data not shown). Based on these findings, we diagnosed the patient with CP-CML.

The patient was administered dasatinib (140 mg/day) as the initial treatment, resulting in a decreasing leukocyte count that gradually returned to normal. The hemoglobin and platelet levels dropped below their normal limits (Fig. 1a, b). A bone marrow aspiration 11 months after the initiation of dasatinib revealed that the patient had not achieved a cytogenetic response in all 20 analyzed mitotic cells, and there was no molecular response (international scale [IS], 103.1\%). We detected a T315I mutation of abl1 by mutational analysis using the bone marrow sample. We then added pegylated interferon alpha (PEG-IFN $\alpha$ ) (90 $\mu \mathrm{g} /$ week) to the dasatinib (100 mg/day). After administering 14 doses of PEG-IFN $\alpha$, we withdrew the drug because the hemoglobin level reached $3.1 \mathrm{~g} / \mathrm{dL}$ (Fig. 1b). The IS value was $38.8 \%$ at that time and increased to $95.7 \%$ at 9 months after the initiation of the dasatinib plus PEG-IFN $\alpha$ combination. Ponatinib was administered instead of dasatinib soon after ponatinib's approval for use for refractory CML. The starting ponatinib dosage was $30 \mathrm{mg} /$ day and was subsequently raised to $45 \mathrm{mg} /$ day. Although we observed no cytogenetic or molecular response since that point, the complete blood counts remained almost within normal limits more than 4 years after starting ponatinib. A few bone marrow aspiration analyses were conducted during the course of treatment, observing a T315I mutation at 9, 25, and 63 months after the start of treatment with dasatinib (Fig. 1b). We detected the chromosomal abnormality $\mathrm{t}(8 ; 9 ; 22)(\mathrm{q} 22 ; \mathrm{q} 34 ; \mathrm{q} 11.2)$ was in 19 of the 20 analyzed mitotic cells at 63 months after the start of treatment with dasatinib; the remaining 1 cell showed a normal karyotype (data not shown).

\section{Karger'}


Table 1. Laboratory data on first admission

\begin{tabular}{ll}
\hline Complete blood counts & Values \\
\hline White blood cells & $298,400 / \mu \mathrm{L}$ \\
Blasts & $8.5 \%$ \\
Promyelocyte & $20.5 \%$ \\
Myelocytes & $11.5 \%$ \\
Metamyelocytes & $3.5 \%$ \\
Stabbed neutrophils & $4.5 \%$ \\
Segmented neutrophils & $37.0 \%$ \\
Lymphocytes & $3.0 \%$ \\
Eosinophils & $5.0 \%$ \\
Basophils & $6.5 \%$ \\
Monocytes & $0.0 \%$ \\
Red blood cells & $231 \times 10^{4} / \mu \mathrm{L}$ \\
Hemoglobin & $7.5 \mathrm{~g} / \mathrm{dL}$ \\
Hematocrit & $22.1 \%$ \\
Platelet & $41.9 \times 10^{4} / \mu \mathrm{L}$ \\
\hline Bone marrow aspiration & \\
\hline
\end{tabular}

Dry tap

Flow cytometry (peripheral mononuclear cells)

\begin{tabular}{lll}
\hline T/NK cell lineage & B cell lineage & Myeloid lineage/others \\
\hline CD2: $0.0 \%$ & CD10: $0.1 \%$ & CD13: $99.1 \%$ \\
CD3: $0.1 \%$ & CD19: $0.0 \%$ & CD14: $0.2 \%$ \\
CD4: $0.5 \%$ & CD20: $0.2 \%$ & CD33: $91.4 \%$ \\
CD5: $1.9 \%$ & & CD34: $84.3 \%$ \\
CD7: $88.8 \%$ & & CD41: $15.5 \%$ \\
CD8: $0.1 \%$ & & CD117: $84.9 \%$ \\
CD56: $4.6 \%$ & & CD235a: $0.6 \%$ \\
& & HLA-DR: $92.4 \%$
\end{tabular}

Chromosomal analysis (peripheral blood)

$46, \mathrm{XY}, \mathrm{t}(8 ; 9 ; 22)(\mathrm{q} 22 ; \mathrm{q} 34 ; \mathrm{q} 11.2)[20]$

bcr-abl1 mRNA analysis (RQ-PCR)

major bcr-abl1 mRNA

minor bcr-abl1 mRNA

$1.1 \times 10^{5}$ copies/ $\mu \mathrm{g}$ RNA

not detected

RQ-PCR, real-time quantitative polymerase chain reaction

To investigate the cellular immunity, T-cell receptor (TCR) Vbeta gene repertoire analyses were performed using flow cytometry targeting CD8-positive T cells at several time points (Fig. 2). We detected fairly high percentages of naïve and effector CTLs using TCR $V$ beta 13.6 at the seventh month after starting dasatinib (naïve CTLs, 33.5\%; effector CTLs, 71.9\%). The percentages of naïve CTLs subsequently showed a gradual increase to approximately $70 \%$, while the percentages of the effector CTLs remained at the same level until the sixty-sixth month after starting dasatinib. Although we observed remarkable clonality of the naïve and 


\section{Changes in leukocyte counts}

$(\mathrm{X} 1,000 / \mu \mathrm{L})$

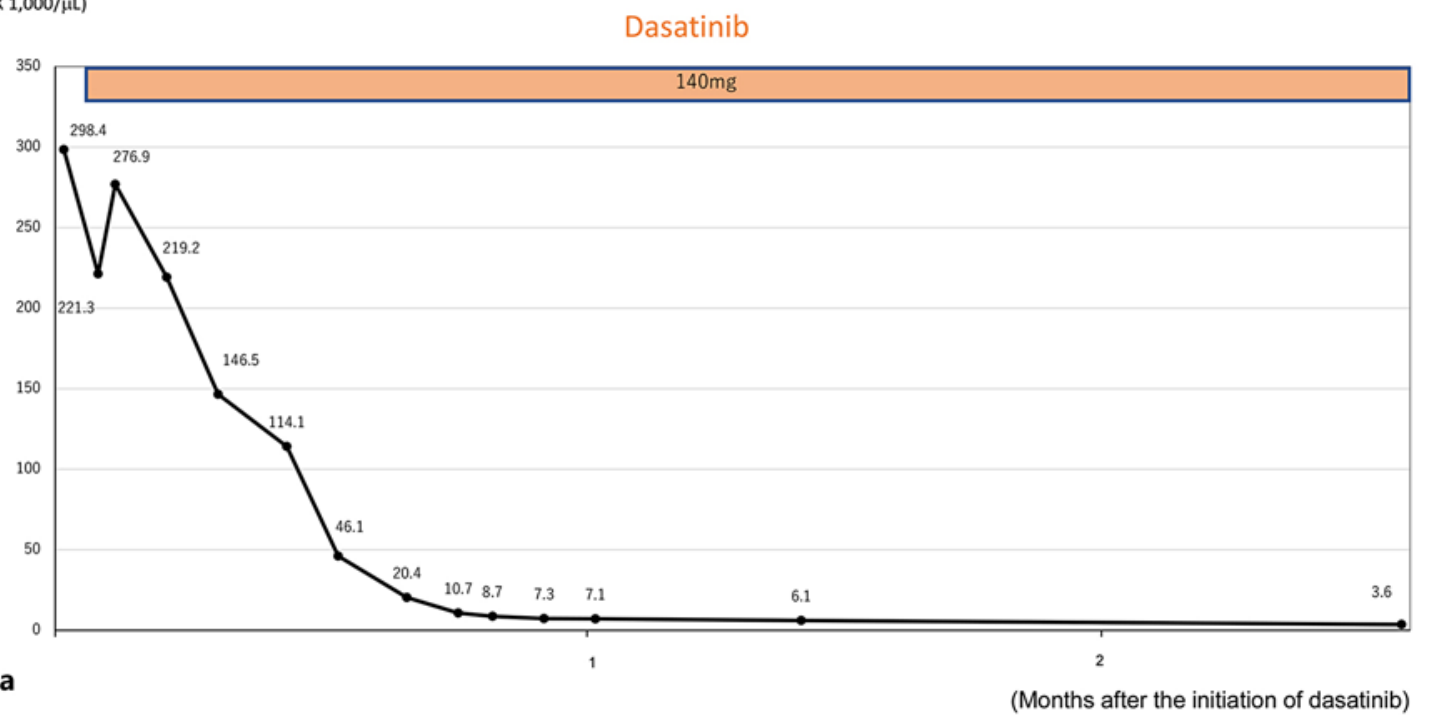

\section{Changes in biomarkers}

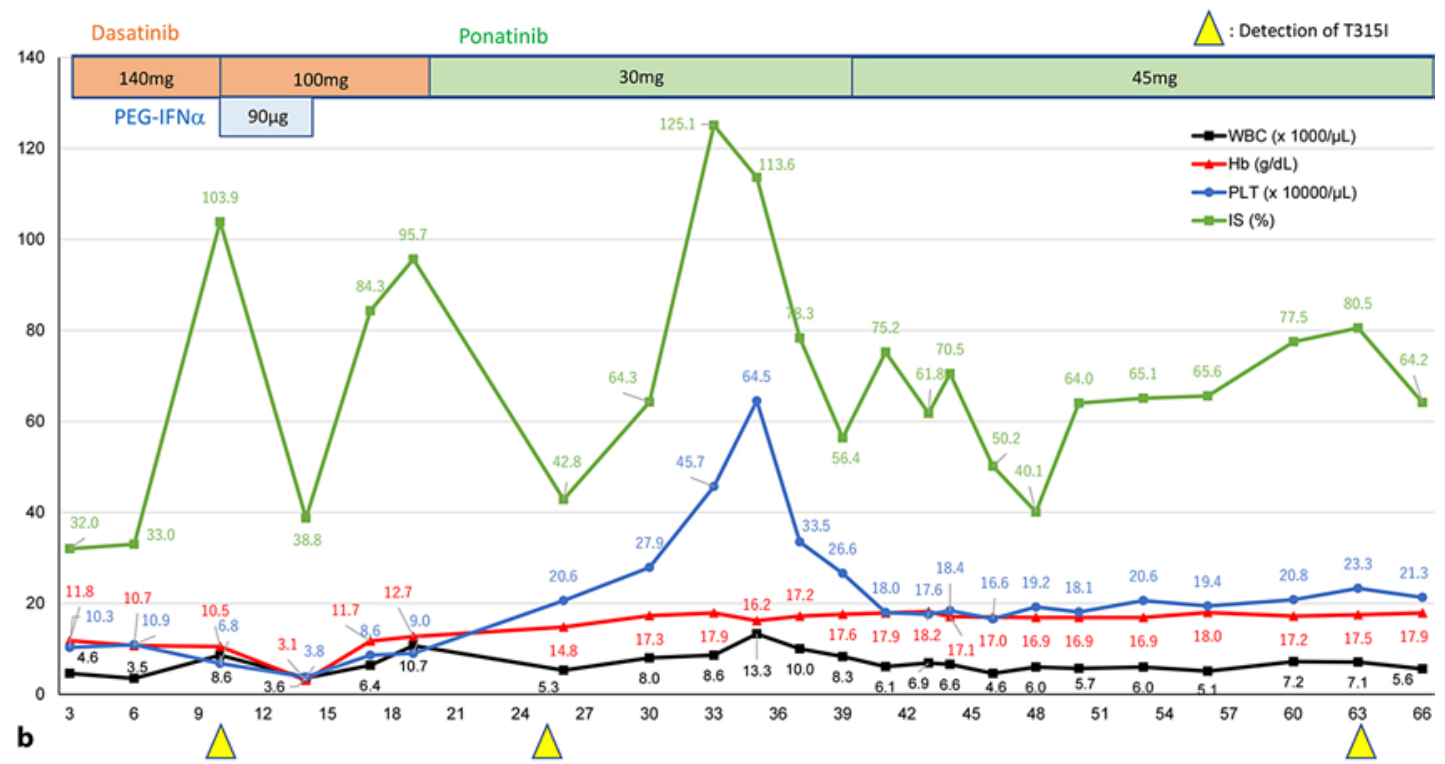

Fig. 1. Changes in the treatment of CML and biomarkers. a Changes in leukocyte counts. $\mathbf{b}$ Changes in biomarkers. Treatment periods and dosages of dasatinib, ponatinib, and pegylated interferon alpha are shown at the top of the figure. The green, black, red, and blue lines indicate the $b c r-a b l 1$ international scale (\%), leukocyte counts $(/ \mu \mathrm{L})$, hemoglobin level $(\mathrm{g} / \mathrm{dL})$, and platelet counts $(/ \mu \mathrm{L})$, respectively. The yellow triangle indicates the time points when the T351I mutation was detected. All data were shown from the third month from start of dasatinib treatment. PEG-IFN $\alpha$, pegylated interferon alpha; WBC, white blood cell; Hb, hemoglobin; PLT, platelet; IS, bcr-abl1 international scale. 


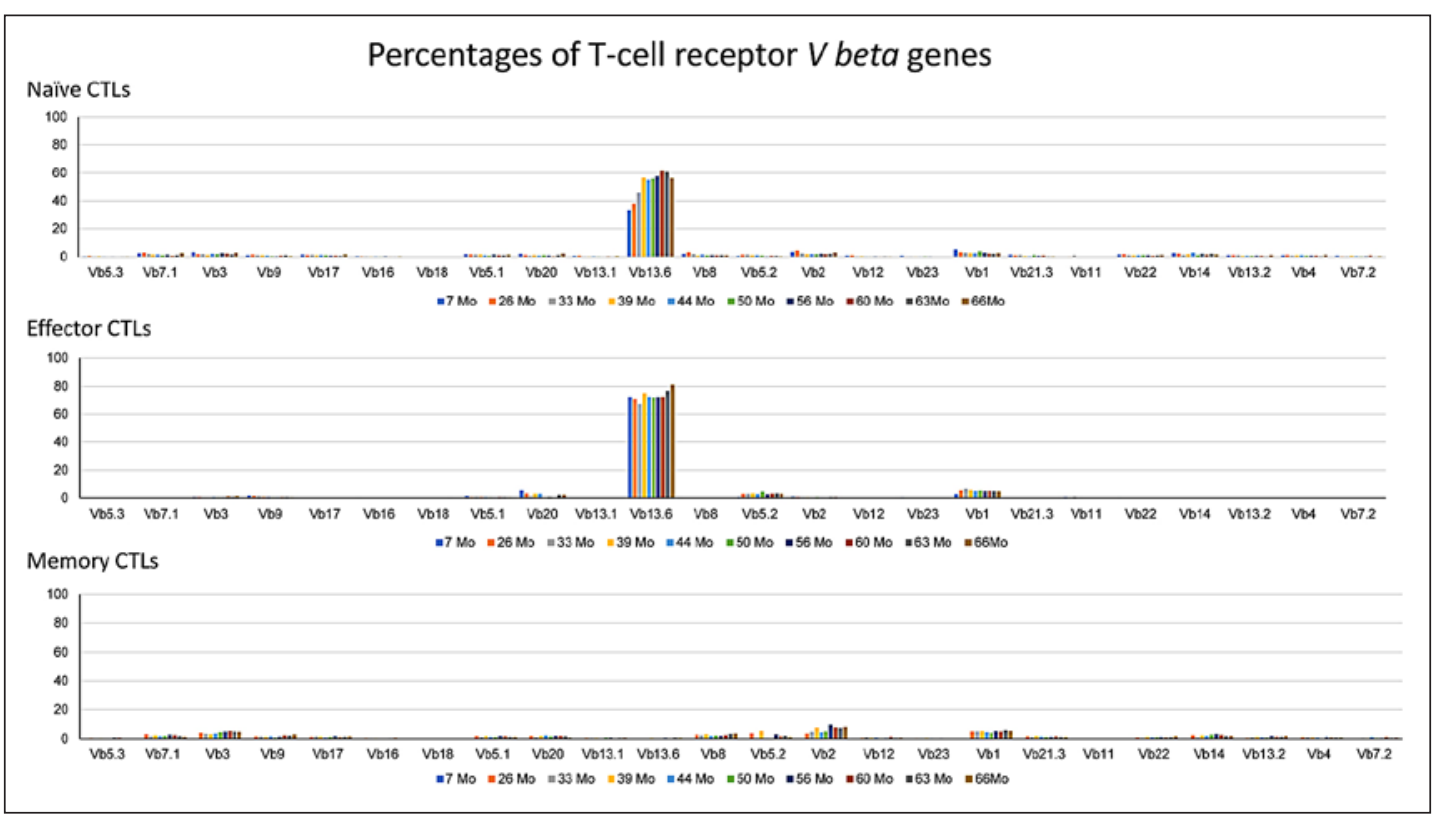

Fig. 2. T-cell receptor $V$ beta gene repertoire analysis. Naïve, effector, and memory CTLs are shown in the upper, middle, and lower panels, respectively. Peripheral blood mononuclear cells were analyzed by flow cytometry using the Beta Mark TCR V $\beta$ repertoire kit (Beckman Coulter, Tokyo, Japan) according to the manufacturer's instructions. Naïve CTLs were defined as the CD8+CD27+CD45RA+ population, effector CTLs were defined as the CD8+CD27-CD45RA+ population, and memory CTLs were defined as the CD8+CD45RApopulation. Vb, $V$ beta; Mo, months after starting dasatinib.

effector CTLs, there has been no obvious clonal expansion of memory CTLs until the present (Fig. 2).

Written informed consent for use and disclosure of the protected information was obtained from the patient.

\section{Discussion/Conclusion}

TKI induction to treat CP-CML has drastically changed the natural course of patients with CP-CML [1], most of whom can expect a normal life expectancy if treated appropriately [2]. However, abl1 mutations often show resistance to treatment with first- and second-generation TKIs such as imatinib, dasatinib, nilotinib, and bosutinib [3-7]. The T315I mutation is especially resistant to all first- and second-generation TKIs. Ponatinib, a third-generation TKI, can overcome the T315I mutation [8]. Regrettably, however, neither a cytogenetic nor molecular response was achieved when this patient was treated with ponatinib. The patient might have had the T315I mutation at the first diagnosis due to the following reasons: (1) there was an additional chromosomal abnormality with $\mathrm{Ph} 1, \mathrm{t}(8 ; 9 ; 22)(\mathrm{q} 22 ; \mathrm{q} 34 ; \mathrm{q} 11.2),(2)$ the initial treatment with dasatinib failure, and (3) the T315I mutation was detected 7 months after starting dasatinib. Generally, patients with CP-CML who are not treated with TKIs will progress to accelerated or blast crisis phases of the disease within 3-5 years. However, our patient spent a normal life with no complaints in the CP phase for more than $5 \frac{1}{2} 2$ years, with no cytogenetic or molecular response. Cellular immunity can play an important role in controlling the disease status of CML. Allogeneic donor lymphocyte infusion in patients with 
relapsed CML after allogeneic hematopoietic stem cell transplantation often shows effectiveness through cellular immunity [9]. In a previous study, we reported that long-lasting effector and memory CTLs with TCR clonality were detected in a patient with CP-CML who could achieve long-term survival with TKI-treatment-free remission and in a patient with Ph1-positive acute lymphoblastic leukemia with long-term complete molecular response [10, 11]. These effector and memory CTLs might play important roles in attacking leukemic stem cells and inhibiting regrowth. We detected the T315I mutation in the early stage of the disease and observed refractoriness against ponatinib in this patient. Nonetheless, the patient was able to survive for more than $5 \frac{1}{2}$ years, living a normal life even with no cytogenetic or molecular response. The clonal expansion of effector CTLs with TCR V beta 13.6 might prevent the patient's CML stem cells with the T315I mutation from transforming into accelerated or blast crisis phases of the disease. However, the effector CTLs were unbale to eradicate the patient's CML stem cells and must therefore have not been able to become memory CTLs so far.

In summary, we report an impressive case of a patient with dasatinib and ponatinibrefractory CP-CML who lived a normal life for more than $5 \frac{1}{2}$ years despite a positive T315I mutation. Expanded effector CTLs with TCR $V$ beta 13.6 might have profoundly contributed to stabilizing the disease status in the chronic phase. Based on our previous reports, cellular immunity against leukemic stem cells with $b c r-a b l 1$ may be a key factor in treating CML and Ph1-positive leukemia.

\section{Acknowledgement}

The authors would like to thank Ms. Riyo Matsumoto for editing the manuscript.

\section{Statement of Ethics}

Written informed consent to publish the content of this case report was obtained from the patient concerned.

\section{Conflict of Interest Statement}

The authors have no conflicts of interest to declare.

\section{Funding Sources}

There was no funding to prepare the manuscript.

\section{Author Contributions}

T.J. designed the study and wrote the original manuscript. T.J. acquired, analyzed, and interpreted the data. T.S., K.M., K.N., and S.H. conducted the data acquisition and the analysis. M.M. and J.T. discussed the conception of the study. All authors helped write the final manuscript.

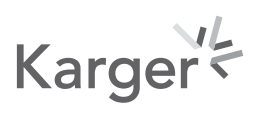




\section{Case Reports in Oncology}

\begin{tabular}{l|l}
\hline Case Rep Oncol 2021;14:493-499 \\
\hline DOI: 10.1159/000514631 & $\begin{array}{l}\text { ○ 2021 The Author(s). Published by S. Karger AG, Basel } \\
\text { www.karger.com/cro }\end{array}$ \\
\hline
\end{tabular}

Jo et al.: Effector CTLs Preventing Blast Crisis in a TKI-Refractory CML Patient

\section{References}

1 Soverini S, Mancini M, Bavaro L, Cavo M, Martinelli G. Chronic myeloid leukemia: the paradigm of targeting oncogenic tyrosine kinase signaling and counteracting resistance for successful cancer therapy. Mol Cancer. 2018;17(1):49.

2 Bower H, Björkholm M, Dickman PW, Höglund M, Lambert PC, Andersson TM. Life expectancy of patients with chronic myeloid leukemia approaches the life expectancy of the general population. J Clin Oncol. 2016;34(24): 2851-7.

3 Gorre ME, Mohammed M, Ellwood K, Hsu N, Paquette R, Rao PN, et al. Clinical resistance to STI-571 cancer therapy caused by BCR-ABL gene mutation or amplification. Science. 2001;293(5531):876-80.

4 Nicolini FE, Corm S, Lê QH, Sorel N, Hayette S, Bories D, et al. Mutation status and clinical outcome of 89 imatinib mesylate-resistant chronic myelogenous leukemia patients: a retrospective analysis from the French intergroup of CML (Fi(phi)-LMC GROUP). Leukemia. 2006;20(6):1061-6.

5 Müller MC, Cortes JE, Kim DW, Druker BJ, ERben P, Pasquini R, et al. Dasatinib treatment of chronic-phase chronic myeloid leukemia: analysis of responses according to preexisting BCR-ABL mutations. Blood. 2009; 114(24):4944-53.

6 Hughes T, Saglio G, Branford S, Soverini S, Kim DW, Müller MC, et al. Impact of baseline BCR-ABL mutations on response to nilotinib in patients with chronic myeloid leukemia in chronic phase. J Clin Oncol. 2009;27(25): 4204-10.2009

7 Khoury HJ, Cortes JE, Kantarjian HM, Gambacorti-Passerini C, Baccarani M, Kim DW, et al. Bosutinib is active in chronic phase chronic myeloid leukemia after imatinib and dasatinib and/or nilotinib therapy failure. Blood. 2012;119(15):3403-12.

8 Cortes JE, Kim DW, Pinilla-Ibarz J, Coutre PL, Paquette R, Chuah C, et al. A phase 2 trial of ponatinib in Philadelphia chromosome-positive leukemias. N Engl J Med. 2013;369:1783-96.

9 Kolb HJ, Schattenberg A, Goldman JM, Hertenstein B, Jacobsen N, Arcese W, et al. Graft-versus-leukemia effect of donor lymphocyte transfusions in marrow grafted patients. European Grouo for Blood and Marrow Transplantation Working party Chronic Leukemia. Blood. 1995;86:2041-50.

10 Jo T, Noguchi K, Hayashi S, Irie S, Hayase R, Shioya H, et al. Long-lasting memory of cellular immunity in a chronic myeloid leukemia patient maintains molecular response 5 after cessation of dasatinib. Oncol Lett. 2018;15(3):2935-8.

11 Jo T, Shioya H, Tominaga H, Sakai T, Hayashi S, Matsuzaka K, et al. Induction of Effector and Memory Cellular Immunity in a Patient with Long-Term Complete Molecular Response to Philadelphia Chromosome-Positive Acute Lymphoblastic Leukemia. Case Rep Oncol. 2020 May-Aug;13(2):990-6. 